\section{State of the art lecture: Endoscopic ultrasound (EUS) and FNA in pancreatico-biliary tumors}

K. J. Chang

University of California, Irvine

\section{Introduction}

In the past few decades, there has been relatively little change in the epidemiology, therapy, or over-all survival of pancreaticobiliary tumors. However, we are now in an era where diagnostic and therapeutic paradigms are finally going beyond traditional approaches (cytology/pathology for diagnosis and cytotoxic agents for therapy) with the potential of changing the natural history of these tumors. Many of these new approaches are made possible with emerging diagnostic and interventional EUS techniques. This state-of-the-art paper will review the role of EUS-guided FNA in the diagnosis of pancreaticobiliary lesions such as pancreatic tumors, cystic and neuroendocrine tumors of the pancreas, as well as the less appreciated applications to biliary and ampullary cancers. I will also discuss the specific role of EUS-guided FNA in the staging of various pancreaticobiliary cancers. The role of EUS-guided therapies, including celiac neurolysis, cyst-gastrostomy, and delivery of anti-tumor agents via EUSguided fine needle aspiration (FNI) will be covered in the accompanying article "FNI and Anti-tumor therapy".

Correspondence: Kenneth J. Chang, M.D. · University of California, Irvine · Director, H. H. Chao Comprehensive Digestive Disease Center · 101 The City Drive, Bldg 22C, RT99 · Orange, CA 92868 - Phone: (714) 456-6187 · Fax: (714) 4567520·E-mail: kchang@uci.edu

Bibliography: Endoscopy 2006; 38 (S1): S56-S60 @ Georg Thieme Verlag KG Stuttgart · New York · ISSN 0013-726X · DOI 10.1055/s-2006-946654

\section{Pancreatic cancer}

Adenocarcinoma is the fifth leading cause for cancer-related death in the United States. Despite improvements in medical and surgical therapy, the overall 5 -year survival still remains at $4 \%$. The most favorable outcome is among surgical patients with small tumors without nodal, vascular, or systemic metastasis. These patients have 5-year survivals that range up to $25 \%$. Optimally, earlier detection and precise pre-operative staging would best stratify patients who would most likely benefit from surgery while sparing the remaining patients from exploratory or palliative-only surgery. Endoscopic ultrasound is considered as one of the most useful diagnostic procedures among the body imaging tools for detecting pancreatic cancer. EUS was shown to be superior (sensitivity $98 \%$ ) to other imaging modalities, including CT, in 146 patients with pancreatic cancer [1]. With the more recent introduction of spiral CT with dual phase contrast, the detection rate for CT is improving. However, recent comparisons between dual-phase spiral CT and endoscopic ultrasound still favor EUS. The ability to obtain cytological specimen by EUS-guided FNA has overcome the difficulty in differentiating between benign vs malignant lesion seen on EUS alone. The application of EUS-guided FNA to the pancreas in particular has great clinical utility. CT or US guided percutaneous FNA are the more common methods for diagnosing pancreatic cancer. The sensitivity of percutaneous FNA ranges from $45 \%$ to $100 \%$, with a specificity of up to $100 \%$. However, obtaining a tissue diagnosis with $\mathrm{CT}$ or US guidance is limited by the ability to visualize the lesion. In a previous multicenter trial, $56 \%$ of patients with pancreatic carcinoma had CT scans which did not demonstrate a mass or revealed nonspecific enlargement of the pancreas [2]. ERCP with cytologic brushing also has a relatively low yield, with sensitivities between $30 \%$ and $56 \%$. The over-all sensitivity, specificity, diagnostic accuracy, NPV and PPV of EUS-guided FNA for pancreatic cancer was $83 \%$, $90 \%, 85 \%, 80 \%$ and $100 \%$. This was superior to CT alone (without FNA): $56 \%, 37 \%, 50 \%, 28 \%$ and $65 \%$, respectively ( $<<0.05)$. There were 4 complications in 164 patients (2\%), including 2 major (perforation, bleeding) and 2 minor (fever). Comparison among the four centers showed that institutions in which a cytologist was present during the procedure had a significantly higher number of passes, cytologic yield, sensitivity and diagnostic accuracy. Advantages of EUS-guided FNA include procuring a tissue diagnosis while also obtaining additional TN staging information the avoidance of additional diagnostic testing and/or surgery and the prognostic information relating to accurate TN staging. In another report from a large single-institution study of 144 pancreatic lesions undergoing EUS-guided FNA, showed a sensitivity, specificity, and diagnostic accuracy of $82 \%, 100 \%$, and $85 \%$ respectively [3]. The most difficult diagnosis to make for any imaging test, including EUS-guided FNA, is the differentiation between pancreatic carcinoma and chronic pancreatitis. Contrast enhanced EUS may improve this differentiation with a recent paper showing the sensitivity increasing from $73 \%$ to $91 \%$ and specificity increasing from $83 \%$ to $93 \%$ with the addition of SonoVue intravenous constrast and power Doppler scanning [4]. With FNA the positive predictive value is almost $100 \%$, although a negative FNA is about $85 \%$ accurate in the setting of pancreatitis. Positron emission tomography (PET) with or without CT fusion may play a role in distinguishing pancreatic cancer from chronic 
pancreatitis $[5,6]$, although results are somewhat disparaging [7-9]. EUS-guided FNA of pancreatic lesions is also worthwhile in patients with a prior negative tissue diagnosis by ERCP or CT of the abdomen. Gress et al. reported his experience with EUSguided FNA of pancreatic mass lesion in 102 patients who had negative cytological tissue diagnosis by ERCP sampling or CTguided FNA [10]. Among those patients, 57 of the 61 patients (93.4\%) with a final diagnosis of pancreatic cancer had positive cytology results for adenocarcinoma by EUS-guided FNA. The false positive results were zero.

There have been several papers written regarding the clinical and economic outcomes of EUS/FNA. One single center study compared the management and survival of 136 patients with pancreatic cancer between the pre and post EUS eras [11]. EUS detected carcinomas that were either not seen or only possibly seen by $\mathrm{CT}$ in $34 \%$ and there were $75 \%$ fewer required operations for diagnosis. The median survival without liver metastases was also longer during the EUS period (102 versus 205 days; $p<0.02$, log-rank test) probably attributable, in part, to lead-time bias. The economic impact of EUS-guided FNA was addressed in an earlier series of 44 consecutive patients who underwent EUS with or without FNA as part of their pancreatic cancer evaluation [12]. Surgery and further diagnostic testing were avoided in $41 \%$ and $57 \%$ of patients respectively. A substantial cost saving of \$ 3300 per patient was calculated. In a series of 216 consecutive patients, the use of EUS with EUS-guided FNA as the initial approach to patients with obstructive jaundice was studied by Erickson et al [13]. EUS/FNA proved useful not only as a diagnostic and staging modality, but also served in directing the need for subsequent therapeutic endoscopic retrograde cholangiopancreatography (ERCP), saving approximately $\$ 1007$ to $\$ 1313$ per patient. In addition, if EUS/ EUS-guided FNA were not utilized at all, an extra $\$ 2200$ would be spent per patient. EUS-guided FNA of the pancreas unlike CT-guided FNA can be preformed during the initial endosonographic procedure. The overall complication rate of EUS-guided FNA was reported to be $0.5 \%-2.9 \%$.

We believe that all patients thought to have operable disease based on initial CT imaging should undergo EUS \pm FNA prior to surgical intervention (see Clinical Algorithm in Fig.1). At the same time, considering the possibility of a false negative result (up to

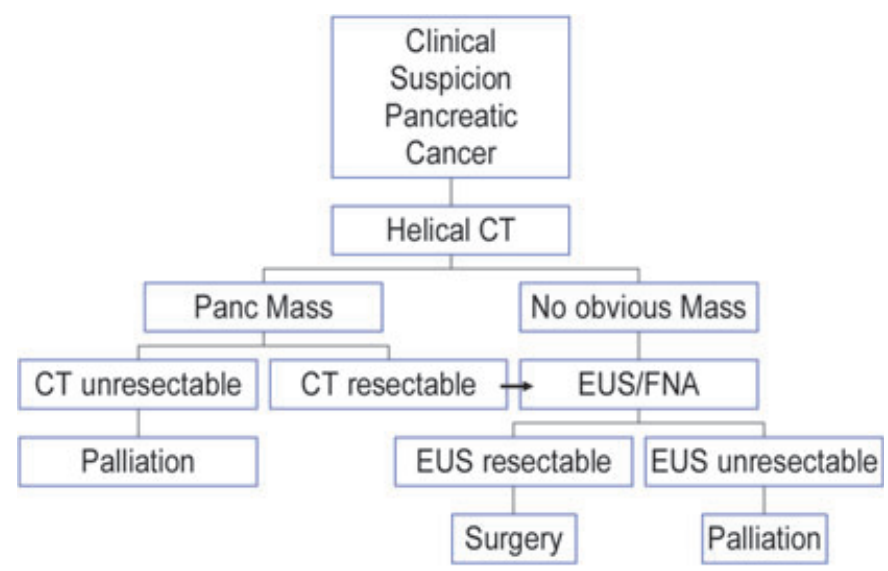

Fig. 1 Algorithm for Diagnosis and Staging of Pancreatic Cancer.
$20 \%$, especially in the setting of chronic pancreatitis), we believe that surgical intervention should not be precluded in a patient with a high suspicion of resectable pancreatic carcinoma and a negative FNA cytology.

In the near future, EUS/FNA will go beyond cytology diagnosis to assess for molecular and/or genetic alterations within the tumor tissue. This is now possible with such techniques as CDNA microarrays which can screen for hundreds of genes simultaneously [14]. Using cDNA microarray, investigators can evaluate specific tumors for chemoresistance-related genes. One such study will have been presented at this year's DDW [15].

\section{Cystic neoplasms}

EUS can be helpful in distinguishing cystic neoplasms from pancreatic pseudocyst, although even here, the specificity is not perfect [16]. The more problematic discernment is between serous and mucinous cysts, with the latter considered pre-malignant. The interobserver agreement for the interpretation of cystic lesions in the pancreas is quite low. The interobserver agreement on 31 pancreatic cyst cases among eight expert endosonographers was shown to be "fair" between endosonographers for diagnosis of neoplastic versus non-neoplastic lesions (kap$\mathrm{pa}=0.24)$ [17]. Agreement for individual types of lesions was moderately good for serous cystadenomas (kappa $=0.46$ ) but fair for the remainder. Accuracy rates of EUS for the diagnosis of neoplastic versus non-neoplastic lesions ranged from $40 \%$ to $93 \%$. Thus, EUS imaging alone is often inadequate for the clinical management of these patients. EUS-guided FNA of cystic contents can be analyzed for cytology, biochemistry and tumor markers. Since cytology is a relatively insensitive test, cyst fluid tumor markers such as CEA have been employed to improve the sensitivity for the detection of malignancy. Cyst fluid CEA values are uniformly low in serous cystadenomas, higher in mucinous lesions, and markedly elevated in mucinous cystadenocarcinomas [18]. A recent cooperative group study examined a battery of tumor markers from pancreatic cysts (CEA, CA 72 - 4, CA 125, CA 19-9, and CA 15-3) among 341 patients (112 with surgical resection). The final diagnosis of the cystic lesions were 68 mucinous, 7 serous, 27 inflammatory, 5 endocrine, and 5 other. Receiver operator curve analysis of the tumor markers demonstrated that cyst fluid CEA (optimal cutoff of $192 \mathrm{ng} / \mathrm{mL}$ ) demonstrated the greatest area under the curve (0.79) for differentiating mucinous vs. nonmucinous cystic lesions. The accuracy of CEA (88 of $111,79 \%$ ) was significantly greater than the accuracy of EUS morphology ( 57 of $112,51 \%$ ) or cytology ( 64 of 109, 59\%) (P $<0.05)$. There was no combination of tests that provided greater accuracy than CEA alone $(\mathrm{P}<0.0001)$. We routinely send cyst fluid for cytology, amylase and CEA. Pseudocysts have very high amylase levels, often over 50,000 IU/L, with a normal CEA and benign cytology. Serous cystadenomas will usually have benign cytology, normal CEA and amylase. Mucinous cystadenomas usually differ from serous cystadenoma in having a high CEA. Mucinous cystadenocarcinoma classically will have malignant cytology, a low amylase, and a markedly elevated CEA. Whereas the cytology from the fluid of malignant cysts may be non-diagnostic. We have found that targeting any solid component, including the cyst wall, may enhance the yield on FNA cytology [19]. 
Endocrine tumors

EUS is very accurate in the detection of neuroendocrine tumors of the pancreas.

Zimmer et al reported their results in localizing and staging neuroendocrine tumors of the foregut in 40 patients examined by EUS, somatostatin receptor scintigraphy (SRS), computed tomography $(\mathrm{CT})$, magnetic resonance imaging (MRI) and transabdominal ultrasound (US) [20]. EUS showed the highest sensitivity in localizing insulinomas compared with SRS, US, CT and MRI. They suggested that US and EUS should be the first-line diagnostics if insulinoma has been proven by a fasting test. Further diagnostic procedures were unnecessary in most cases. Further diagnostics such as CT or MRI to search for distant metastases are necessary in large tumors or local invasive tumors. For gastrinomas, EUS has the highest accuracy to detect or exclude pancreatic gastrinomas, but fails to detect extrapancreatic gastrinomas in about $50 \%$. The combination of EUS and SRS may give complementary information. They recommended that the first-line diagnostics in gastrinoma patients should be SRS and CT or MRI. If no metastases are detected, EUS should be the next preoperative imaging procedure. In nonfunctional neuroendocrine tumors, EUS provides the best information on local tumor invasion and regional lymph node involvement. EUS has also been shown to be cost effective in the preoperative localization of pancreatic endocrine tumors [21] largely because of reductions in the number of diagnostic angiograms and venous sampling procedures performed. The more specific utility of EUS-guided FNA in these patients was recently reported [22] and shown to be helpful. In addition, EUS may also be useful in marking these subtle lesions using EUS-guided fine needle "tattooing" prior to surgery to assist in intra-operative localization [23].

\section{EUS-guided FNA in the staging of pancreatic cancer}

Although EUS has historically been superior to CT or MR in staging pancreatic cancer, this may no longer be the case. A recent review analyzed the pooled data among 4 comparative studies and still showed superiority of EUS to helical CT in detection of pancreatic tumor (97\% vs $73 \%$ ), in accuracy for respectability (91\% vs $83 \%$ ), and sensitivity for vascular invasion (91\% vs 64\%) [24]. However, with even newer imaging modalities such as contrast-enhanced multi-detector row helical CT (MDR-CT), which allows for 3-dimensional maximum intensity projection (MIP) and volume rendered images (VRI), the vascular staging of pancreatic cancer by CT will likely surpass that of EUS [25,26]. In addition, the fusion of CT with PET may give additional distant staging information. However, EUS-guided FNA will continue to play an important role for staging pancreatic cancer in sampling metastatic lymph nodes, liver lesions, and ascites. The economic impact of EUS-guided FNA in the preoperative staging of patients with pancreatic head adenocarcinoma was clearly demonstrated in a decision analysis model [27]. The use of EUS-guided FNA prevented 16 surgeries per 100 patients compared to 8 per 100 patients if CT-guided FNA was performed for non-peritumoral lymph nodes. If the frequency of non-peritumoral lymph nodes was $>4 \%$ then EUS-guided FNA is the least costly procedure ( $\$$ $15,938)$ vs $(\$ 16,378)$ for CT-FNA and $(\$ 18,723)$ for surgery. According to a multivariate analysis, lymph node metastasis, intra- pancreatic perineural invasion, and portal vein invasion are significant prognostic factors in patients with pancreatic cancer after curative resection [28]. In a multi-center study 171 patients underwent EUS-gduied FNA of 192 lymph nodes (46 benign, 146 malignant) [29]. When comparing EUS-guided FNA with EUS size criteria ( $£ 10 \mathrm{~mm}=$ benign), the sensitivity ( $90 \%$ versus $91 \%, \mathrm{P}=$ n.s.) and accuracy (92\% versus $83 \%, \mathrm{P}=$ n. s.) for EUSguided FNA were similar whereas the specificity was superior to that of EUS size criteria alone (100\% versus $47 \%, \mathrm{p}<.001)$. Not only can EUS-guided FNA improve the specificity of lymph node metastasis with cytologic confirmation, most recently FNA has been used to detect genetic alterations in cytologic negative nodes [30]. Although there was no significant difference in overall survival rates between the pathological node-negative and -positive patients, overall survival of the patients with nodes-negative for the mutated K-ras gene were significantly better than that of the patients with genetically metastasis-positive nodes ( $p<0.001$ ). These findings suggest that detection of K-ras gene mutations in lymph nodes may be clinically useful to stratify patients who may be at higher risk for recurrence after curative resection.

\section{Liver metastasis}

EUS was not traditionally thought to be clinically applicable in imaging the liver imaging. However, both prospective [31] and retrospective [32] studies have shown the ability of EUS to detect lesions not seen on CT and the ability to safely perform EUS-guided FNA on the same procedure. EUS-guided FNA should be considered when a liver lesion is poorly accessible to percutaneous FNA or when US or CT-guided FNA fail to make a diagnosis. If EUS detects a liver lesion de novo in the setting of staging pancreatic cancer, EUS-guided FNA should be attempted first, even prior to taking biopsies of the primary pancreatic tumor. Liver lesions have a much higher cytologic yield (less needle passes requires, less inflammatory and fibrotic reaction) and give the highest staging information.

\section{Ascites}

The utility of EUS/FNA was evaluated for detection and aspiration of scant ascites among patients undergoing EUS for diagnosis and staging of GI malignancies [33]. Eighty-five patients (15\% of a series of 571 patients) were found to have ascites by EUS. PreEUS CT identified ascites in only $18 \%$ of patients with ascites on EUS. 31 of the 85 patients underwent EUS-guided FNA paracentesis and in 5 patients, malignant ascites was diagnosed by EUSguided FNA. The clinical impact was high in these patients as surgery was avoided.

\section{EUS-guided FNA in biliary lesions}

\section{Diagnosis and staging of cholangiocarcinoma}

EUS-guided FNA is now being used to diagnose and stage cholangiocarcinoma. In one case series, 10 patients with bile duct strictures at the hepatic hilum, diagnosed by CT and/or ERCP, underwent EUS-guided FNA. Adequate material was obtained in nine patients [34]. Cytology revealed cholangiocarcinoma in seven and hepatocellular carcinoma in one. One benign inflammatory lesion identified on cytology proved to be a false-negative finding by frozen section. Metastatic locoregional hilar lymph nodes were detected in two patients, and in one patient the celiac and 
para-aortic lymph nodes were aspirated to obtain tissue proof of distant metastasis. In a retrospective series of 238 patients with suspected or known biliary strictures, EUS-guided FNA obtained a tissue diagnosis in 12/26 (46\%) patients, which were negative on cytology, or had a unsuccessful ERCP [35]. There were no complications. These studies suggest that EUS with FNA is safe, and effective in evaluating proximal biliary strictures. When used in combination with ERCP, it helps distinguish benign from malignant strictures, and facilitates a definitive diagnosis by increasing tissue yield.

\section{Diagnosis and staging of ampullary cancer}

Conventional abdominal imaging studies such as CT, MRI, and transabdominal ultrasound frequently fail to detect ampullary lesions. EUS is a sensitive modality for detecting and staging ampullary tumors. Accurate staging may be affected by biliary stenting, which is frequently performed in these patients with obstructive jaundice. Combined data from two centers reported the accuracy of ampullary tumor staging with multiple imaging modalities in patients with and those without endobiliary stents [36]. Fifty consecutive patients with ampullary neoplasms were preoperatively staged by EUS plus CT (37 patients), MRI (13 patients), or angiography (10 patients) over a 3.5 year period. Twenty-five of the 50 patients had a transpapillary endobiliary stent present at the time of endosonographic examination. EUS was shown to be more accurate than CT and MRI in the overall assessment of the T stage of ampullary neoplasms (EUS 78\%, CT $24 \%$, MRI $46 \%$ ). No significant difference in N stage accuracy was noted between the three imaging modalities (EUS 68\%, CT $59 \%$, MRI 77\%). EUS T stage accuracy was reduced from $84 \%$ to $72 \%$ in the presence of a transpapillary endobiliary stent. This was most prominent in the understaging of T2/T3 carcinomas. More recently, a retrospective study was published in which the role of EUS-guided FNA in the diagnosis and staging of ampullary lesions has reported [37]. EUS-guided FNA was performed in 20 of 27 (74\%) patients with suspected ampullary tumors. EUS-guided FNA made the initial ampullary tissue diagnosis in 7 patients (adenocarcinoma-5, adenoma-1, neuroendocrine tumor-1). In addition, EUS-guided FNA resulted in a change of the diagnosis from adenoma to adenocarcinoma in one patient. In 1 patient, EUS-guided FNA diagnosed a liver metastasis not seen on CT. Overall, EUS-guided FNA provided new histologic information in $9 / 27$ patients (33\%).

\section{Conclusions}

EUS-guided FNA is extremely useful in the diagnosis and staging of pancreaticobiliary lesions such as pancreatic cancers, cystic tumors, neuroendocrine neoplasms, ampullary and cholangiocarcinomas. In addition, this technique has now been extended to therapeutic modalities such as celiac nerve block, cyst-gastrostomy and delivery of anti-tumor agents (see associated article on FNI).

\section{References}

${ }^{1}$ Rosch T, Lorenz R, Braig C, Feuerbach S, Siewert JR, Schusdziarra V et al. Endoscopic ultrasound in pancreatic tumor diagnosis. Gastrointest Endosc 1991; 37 (3): $347-352$
${ }^{2}$ Chang KJ, Wiersema M, Giovannini M, Vilmann P, Erickson RA. Multicenter Collaborative Study on Endoscopic Ultrasound (EUS) Guided Fine Needle Aspiration (FNA) of the Pancreas. Gastrointest Endosc 1996; 43 (4): A507

${ }^{3}$ Williams DB, Sahai AV, Aabakken L, Penman ID, van Velse A, Webb J et al. Endoscopic ultrasound guided fine needle aspiration biopsy: a large single centre experience. Gut 1999; 44 (5): 720-726

${ }^{4}$ Hocke M, Schulze E, Gottschalk P, Topalidis T, Dietrich CF. Contrast-enhanced endoscopic ultrasound in discrimination between focal pancreatitis and pancreatic cancer. World J Gastroenterol 2006; 12 (2): 246-250 ${ }^{5}$ van Kouwen MC, Jansen JB, van Goor H, de Castro S, Oyen WJ, Drenth JP. FDG-PET is able to detect pancreatic carcinoma in chronic pancreatitis. Eur J Nucl Med Mol Imaging 2005; 32 (4): 399-404

${ }^{6}$ Heinrich S, Goerres GW, Schafer M, Sagmeister M, Bauerfeind P, Pestalozzi BC et al. Positron emission tomography/computed tomography influences on the management of resectable pancreatic cancer and its cost-effectiveness. Ann Surg 2005; 242 (2): 235-243

${ }^{7}$ Lytras D, Connor S, Bosonnet L, Jayan R, Evans J, Hughes M et al. Positron emission tomography does not add to computed tomography for the diagnosis and staging of pancreatic cancer. Dig Surg 2005; 22 (12): $55-61$; discussion 62

${ }^{8}$ Rasmussen I, Sorensen J, Langstrom B, Haglund U. Is positron emission tomography using $18 \mathrm{~F}$-fluorodeoxyglucose and $11 \mathrm{C}$-acetate valuable in diagnosing indeterminate pancreatic masses? Scand J Surg 2004; 93 (3): $191-197$

${ }^{9}$ Orlando LA, Kulasingam SL, Matchar DB. Meta-analysis: the detection of pancreatic malignancy with positron emission tomography. Aliment Pharmacol Ther 2004; 20 (10): 1063 - 1070

${ }^{10}$ Gress F, Gottlieb K, Sherman S, Lehman G. Endoscopic ultrasonography-guided fine-needle aspiration biopsy of suspected pancreatic cancer. Annals of Internal Medicine 2001; 134 (6): 459 - 464

${ }^{11}$ Erickson RA, Garza AA. Impact of endoscopic ultrasound on the management and outcome of pancreatic carcinoma. Am J Gastroenterol 2000; 95 (9): 2248 - 2254

12 Chang KJ, Nguyen P, Erickson RA, Durbin TE, Katz KD. The clinical utility of endoscopic ultrasound-guided fine-needle aspiration in the diagnosis and staging of pancreatic carcinoma. Gastrointestinal Endoscopy 1997; 45 (5): 387-393

${ }^{13}$ Erickson RA, Garza AA. EUS with EUS-guided fine-needle aspiration as the first endoscopic test for the evaluation of obstructive jaundice. Gastrointest Endosc 2001; 53 (4): 475-484

14 Jin G, Hu XG, Ying K, Tang Y, Liu R, Zhang YJ et al. Discovery and analysis of pancreatic adenocarcinoma genes using cDNA microarrays. World J Gastroenterol 2005; 11 (41): 6543-6548

${ }^{15}$ Ashida R, Nakata B, Inoue H, Mizuno N, Higuchi K, Hirakawa K et al. Chemoresistance-related genes profile in specimens obtained by endoscopic ultrasound-guided fine-needle aspiration (EUS-FNA). Gastrointest Endosc, 2006: DDW abstract

${ }^{16}$ Song MH, Lee SK, Kim MH, Lee HJ, Kim KP, Kim HJ et al. EUS in the evaluation of pancreatic cystic lesions. Gastrointest Endosc 2003; 57 (7): $891-896$

${ }^{17}$ Ahmad NA, Kochman ML, Brensinger C, Brugge WR, Faigel DO, Gress $\mathrm{FG}$ et al. Interobserver agreement among endosonographers for the diagnosis of neoplastic versus non-neoplastic pancreatic cystic lesions. Gastrointest Endosc 2003; 58 (1): 59-64

${ }^{18}$ Brugge WR, Lewandrowski K, Lee-Lewandrowski E, Centeno B, Szydlo T, Regan S et al. Diagnosis of pancreatic cystic neoplasms: a report of the cooperative pancreatic cyst study. Gastroenterology 2004; 126 (5): $1330-1336$

${ }^{19}$ Powis ME, Nguyen PT, Chang KJ. A Novel Endoscopic Ultrasound (EUS) Guided Fine Needle Aspiration (FNA) Technique for the Diagnosis of Malignant Cystic Lesions of the Pancreas. Gastrointest Endosc 2000; 51 (4): 164

${ }^{20}$ Zimmer T, Scherubl H, Faiss S, Stolzel U, Riecken EO, Wiedenmann B. Endoscopic ultrasonography of neuroendocrine tumours. Digestion 2000; 62 (Suppl 1): 45-50

${ }^{21}$ Bansal R, Tierney W, Carpenter S, Thompson N, Scheiman JM. Cost effectiveness of EUS for preoperative localization of pancreatic endocrine tumors. Gastrointestinal Endoscopy 1999; 49 (1): 19-25

22 Gines A, Vazquez-Sequeiros E, Soria MT, Clain JE, Wiersema MJ. Usefulness of EUS-guided fine needle aspiration (EUS-FNA) in the diagnosis of functioning neuroendocrine tumors. Gastrointest Endosc 2002; 56 (2): $291-296$ 
${ }^{23}$ Gress FG, Barawi M, Kim D, Grendell JH. Preoperative localization of a neuroendocrine tumor of the pancreas with EUS-guided fine needle tattooing. Gastrointest Endosc 2002; 55 (4): 594-597

${ }^{24}$ Hunt GC, Faigel DO. Assessment of EUS for diagnosing, staging, and determining resectability of pancreatic cancer: a review. Gastrointest Endosc 2002; 55 (2): $232-237$

25 Tamm E, Charnsangavej C, Szklaruk J. Advanced 3-D imaging for the evaluation of pancreatic cancer with multidetector CT. Int J Gastrointest Cancer 2001; $30(1-2): 65-71$

${ }^{26}$ Xu AM, Cheng HY, Jiang WB, Chen D, Jia YC, Wu MC. Multi-slice threedimensional spiral CT cholangiography: a new technique for diagnosis of biliary diseases. Hepatobiliary Pancreat Dis Int 2002; 1 (4): 595 603

${ }^{27}$ Harewood GC, Wiersema MJ. A cost analysis of endoscopic ultrasound in the evaluation of pancreatic head adenocarcinoma. Am J Gastroenterol 2001; 96 (9): $2651-2656$

28 Ozaki H, Hiraoka T, Mizumoto R, Matsuno S, Matsumoto Y, Nakayama $\mathrm{T}$ et al. The prognostic significance of lymph node metastasis and intrapancreatic perineural invasion in pancreatic cancer after curative resection. Surgery Today 1999; 29 (1): 16-22

${ }^{29}$ Wiersema MJ, Vilmann P, Giovannini M, Chang KJ, Wiersema LM. Endosonography-guided fine-needle aspiration biopsy: diagnostic accuracy and complication assessment. Gastroenterology 1997; 112 (4): 1087- 1095

${ }^{30}$ Yamada T, Nakamori S, Ohzato H, Higaki N, Aoki T, Oshima S et al. Outcome of pancreatic cancer patients based on genetic lymph node staging. International Journal of Oncology 2000; 16 (6): 1165-1171

${ }^{31}$ Nguyen P, Chang K. Endoscopic Ultrasound (EUS) and EUS-Guided Fine Needle Aspiration (FNA) of Liver Lesions in Patients with Gastrointestinal Malignancies. Gastrointest Endosc 1999; 50 (3): 357 - 361

32 tenBerge J, Hoffman BJ, Hawes RH, Van Enckevort C, Giovannini M, Erickson RA et al. EUS-guided fine needle aspiration of the liver: indications, yield, and safety based on an international survey of $167 \mathrm{ca}-$ ses. Gastrointest Endosc 2002; 55 (7): 859-862

${ }^{33}$ Nguyen PT, Chang KJ. EUS in the detection of ascites and EUS-guided paracentesis. Gastrointest Endosc 2001; 54 (3): 336-339

${ }^{34}$ Fritscher-Ravens A, Broering DC, Sriram PV, Topalidis T, Jaeckle S, Thonke F et al. EUS-guided fine-needle aspiration cytodiagnosis of hilar cholangiocarcinoma: a case series. Gastrointest Endosc 2000; 52 (4): $534-540$

35 Sharma A, Chang KJ, Nguyen PT. The Role of Endoscopic Ultrasound (EUS) and EUS-guided Fine Needle Aspiration (FNA) in the Diagnosis of Proximal Biliary Strictures. Gastrointest Endosc 2003; 57 (5): AB681

${ }^{36}$ Cannon ME, Carpenter SL, Elta GH, Nostrant TT, Kochman ML, Ginsberg GG et al. EUS compared with CT, magnetic resonance imaging, and angiography and the influence of biliary stenting on staging accuracy of ampullary neoplasms. Gastrointestinal Endoscopy 1999; 50 (1): $27-33$

37 Muthusamy R, Jafri SF, Jivcu C, Chang KJ, Nguyen PT. Endoscopic Ultrasound (EUS) Guided Fine Needle Aspiration (FNA) in the Diagnosis and Staging of Ampullary Neoplasms. Gastrointest Endosc 2001; 53(5): 176 